\title{
Tegaderm transparent dressing (3M) with antibiotic ointment for treatment of infectious corneal ulcer: Antibiotic Wet Therapy
}

Shingo Yasuda ( $\sim$ d_maxwel@wakayama-med.ac.jp )

Wakayama Medical University https://orcid.org/0000-0003-1019-2459

\section{Takayoshi Sumioka}

Wakayama Medical University

\section{Yukihisa Takada}

Wakayama Medical University

\section{Yuka Okada}

Wakayama Medical University

Shizuya Saika

Wakayama Medical University

Original research

Keywords: cornea, infectious corneal ulcer, antibiotic ointment

Posted Date: March 11th, 2021

DOI: https://doi.org/10.21203/rs.3.rs-276979/v1

License: (c) (1) This work is licensed under a Creative Commons Attribution 4.0 International License. Read Full License 


\section{Abstract \\ Purpose}

To retrospectively review cases of infectious corneal ulcer treated with Tegaderm transparent dressing (3M) with an antibiotic ointment.

\section{Subjects and methods:}

A series of 29 eyes of 29 patients with possible infectious corneal keratitis were treated in Wakayama Medical University Hospital between January 1st, 2016 and December 31st, 2018. The age of the patients ranged from 26 to 92 years (mean $68.0 \pm 17.8$ ). At the first visit, the corneal infection lesion was scraped from the epithelium to the substantially superficial layer, and the scraped matter was submitted to Gram staining and culture examination. Cases suspected of fungi and Acanthamoeba were examined directly with potassium hydroxide $(\mathrm{KOH})$ treatment. For several days until drug sensitivity results were obtained, initial treatment was Antibiotic Wet Therapy (AWT) with ophthalmic eye ointment and transparent Tegaderm (3M) dressing. We retrospectively reviewed the culture results of corneal scrapings (detected bacteria and drug resistance) and the therapeutic outcome of AWT.

\section{Results}

Nineteen eyes in which the causative bacteria were not identified and five eyes in which the causative bacteria (Pseudomonas aeruginosa, methicillin-susceptible Staphylococcus aureus (MSSA), Streptococcus, Corynebacterium.) were identified were successfully treated using AWT with ofloxacin ointment. methicillin-resistant Staphylococcus aureus (MRSA) was detected in 3 of 5 eyes in which AWT with ofloxacin was not successful, and filamentous fungi were detected in one eye. Two of the three eyes MRSA detected were healed by changing to vancomycin hydrochloride eye ointment, one of which was severely affected and was later enucleated. One eye with filamentous fungi was treated after changing to pimaricin eye ointment. A causative pathogen was not identified in the other eye, but because it was suspected to be fungal the ointment was changed to a pimaricin eye ointment, which led to healing.

\section{Conclusion}

AWT may be an option for initial treatment of corneal infections when the causative organism is unknown.

\section{Introduction}

It is important that the injured or infected cornea remains transparent after treatment [1-3]. Scarring following wound healing in the corneal stroma results in opacification and irregular astigmatism, both of 
which impair visual function [4, 5]. Therefore, initial treatment of corneal infection is critical for a final outcome of healing with minimal scarring. In a clinical setting, application of a broad-spectrum antibiotic eye drop is the first choice of treatment until bacteriology examination identifies the specific pathogen [6, 7]. For over ten years, we have treated infectious corneal ulcers using Antibiotic Wet Therapy (AWT, an antibiotic ophthalmic ointment with Tegaderm (3M) transparent dressing) as the initial treatment. In this study, we retrospectively reviewed the culture results of corneal scrapings (detected bacteria and drug resistance) and the therapeutic outcomes of AWT in cases from January 1st, 2016 to December 31st, 2018. AWT was successful in most cases, even without identification of a causative pathogen.

\section{Subjects}

This retrospective study was approved by the Ethical Review Board of Wakayama Medical University Hospital (\#2620). The study included a series of 29 eyes of 29 patients with suspected infectious corneal disorder treated in Wakayama Medical University Hospital during the between January 1st, 2016 and December 31st, 2018. Patients were aged 26 to 92 years (mean $68.0 \pm 17.8$ ).

At the first visit, the corneal infection lesion was scraped from the epithelium to the substantially superficial layer, and the scraped matter was submitted to Gram staining and culture examination. Cases suspected of fungi and Acanthamoeba were examined directly with potassium hydroxide $(\mathrm{KOH})$ treatment $[8,9]$.

Pimaricin eye ointment was used to treat confirmed fungal infection. Ophthalmic eye ointment was used for suspected pathogens sensitive to new quinolone, such as Pseudomonas aeruginosa, as the initial treatment for unknown pathogens. When methicillin-resistant Staphylococcus aureus (MRSA) or fungi were detected with culture examination, treatment was changed from ophthalmic ointment to vancomycin hydrochloride or pimaricin ointment.

The AWT method involves applying about $0.5 \mathrm{~g}$ eye ointment in the lower conjunctival sac and affixing Tegaderm transparent dressing (3M) (Figure 1) to discourage eye opening and to develop a tear-filled environment inside the film.

Our hospital policy is to only remove the protective film for treatment, such as for corneal abrasion at examination. This usually occurs once per day, but if the wet state cannot be maintained, additional exchange is performed. We aim to ensure the eye is always moist, but film removal did not exceed 3 times per day if possible to avoid damaging the skin around the eyelid.

\section{Methods}

In this study, we retrospectively reviewed the culture results of corneal scrapings (including bacteria and drug resistance) and the therapeutic outcomes of AWT. The eyes were grouped two [Therapeutic response $(+)$ group and Therapeutic response (-) group], and causative pathogens were assayed in each group. 
Representative case was also shown here. In this patient we did not detect causative specific pathogens were detected, but AWT was successful.

\section{Result}

AWT was successful in the treatment of 19 eyes in which the causative bacteria were not identified and five eyes in which causative bacteria were identified as Pseudomonas aeruginosa, methicillin-sensitive Staphylococcus aureus (MSSA), Streptococcus and Corynebacterium. (Table 1)

Table 1

Number and types of causative pathogens with positive or negative response to AWT.

\begin{tabular}{|lll|}
\hline Therapeutic response + & & 24 \\
\hline & Pseudomonas aeruginosa & 3 \\
\hline MSSA & 1 \\
\hline & Streptococcus & 1 \\
\hline & Corynebacterium & 1 \\
\hline & & $($ Partially duplicated) \\
\hline Therapeutic response - & & 19 \\
\hline & MRSA detection & 5 \\
\hline & fungus & 3 \\
\hline No detection & 1 \\
\hline
\end{tabular}

Of a further five eyes in which AWT had no therapeutic effect, three eyes had MRSA infection, one eye had causative fungus infection and one eye had an unknown cause of infection.

For the three eyes with MRSA infection, ofloxacin ointment was changed to vancomycin ointment and inflammation was improved in two out of three eyes, but one eye with severe MRSA infection required enucleation. The eye with a fungal cause of infection was improved by changing ofloxacin to pimaricin ointment, and in the eye with unknown cause of infection, but suspected to be fungal, the ointment was changed to a pimaricin eye ointment, which led to healing.

In this retrospective study, there was no recurrence of corneal infection.

\section{Representative Case Report}


A 67-year-old male patient complained of left eye pain and decreased visual acuity and was diagnosed with infectious keratitis. Two days later, a corneal ulcer appeared, and he visited our hospital. The findings at the first visit were visual acuity: Right $=0.7$ (1.0) Left $=0.05$ (no improvement with refractive correction). Congestion of the bulbar conjunctiva was prominent, and a central corneal ring-shaped ulcer and inflammatory cells in the anterior chamber were found. (Fig. 2)

As initial treatment, corneal lesion scrubbing and Gram staining was performed, but no causative bacteria were identified. Infectious corneal ulcer was suspected on the basis of clinical findings, and AWT was started. One week later, the genus Streptococcus was detected by culture examination, and sensitivity to levofloxacin was confirmed. The visual acuity on day 18 after the start of AWT was Right $=0.7$ (1.0) and Left $=0.5$ (no improvement with refractive correction). Bulbar conjunctival hyperaemia was reduced and inflammatory cells in the anterior chamber had disappeared. Although corneal opacity remained, epithelial surface was observed and the ulcer had healed. (Fig. 3) The treatment was terminated at this point, but it was noted that the patient had already lapsed treatment without relapse of corneal ulcer or keratitis.

\section{Discussion}

In the current study, 24 of 29 cases were successfully treated using AWT. The efficacy of the treatment strategy might depend on water retention and long-standing exposure of antibiotics to the lesion. The common use of dressings in treatment of cutaneous burn or decubitus $[10,11]$ led us to hypothesize that maintenance of a moist environment may be favourable in the treatment of corneal injury or infection. A wound-healing effect of tear retention might depend on activation of cell behaviours and provision of wound healing-related bioactive components, such as growth factors and extracellular matrix components like fibronectin [12-14].

Physiological tear clearance is calculated as the sum of tear secretion, including tears lost from the conjunctiva, tear evaporation, and tear discharge to the nasolacrimal duct, and occurs at a stable rate. Human tear turnover rate is said to be around $10 \%-20 \%$ per minute $[15,16]$. With frequent eye drops, the quantity of tears temporarily increases, but it is thought that this quantity increases over time and as the tears flow from the ocular surface. By applying Tegaderm to form an enclosed environment, the tears that would have evaporated were retained and accumulated in the space inside the Tegaderm transparent dressing. Antibiotic ointments mixed with tears were also stored inside this environment over a sustained period. Therefore, Tegaderm transparent dressing may have been useful in keeping antibiotics, growth factors and extracellular matrix components in contact with the eye for a long time.

\section{Conclusion}

In the cases reported here we applied ofloxacin ophthalmic ointment prior to obtaining results of bacteriology culture testing. In 19 cases the lesion was successfully treated with AWT treatment even though the bacteriology testing did not identify a specific pathogen. In five cases the bacteria were 
sensitive to ofloxacin with favourable outcome. In five cases with MRSA or fungi, AWT did not yield satisfactory outcomes. However, the drug suitable to these pathogens showed effectiveness. We consider it is not suitable to continue AWT if effectiveness is not seen following several days of treatment. Another treatment strategy for bacterial keratitis is frequent instillation of an antibiotic eye drop. However, AWT treatment involves reduced drug instillation, which may be beneficial to both patient and medical team.

\section{Abbreviations}

AWT

Antibiotic Wet Therapy (AWT)

MSSA

methicillin-susceptible Staphylococcus aureus

MRSA

methicillin-resistant Staphylococcus aureus

\section{Declarations}

\section{Ethics approval and consent to participate}

This retrospective study was approved by the Ethical Review Board of Wakayama Medical University Hospital (\#2620).

\section{Consent for publication}

Written informed consent for publication was obtained.

\section{Availability of data and material}

The datasets used during the current study are available from the corresponding author on reasonable request.

\section{Competing interests}

There is no financial disclosure.

\section{Funding}

Not applicable.

\section{Authors' contributions}

All authors contributed to project conception and critical review of manuscript. The author(s) read and approved the final manuscript. 
Acknowledgements

Not applicable.

\section{Authors' information (optional)}

Not applicable.

\section{References}

1. Brayden B, Sarah E, Amy W et al (2019) Corneal injury: Clinical and molecular aspects. Exp Eye Res 186:107709

2. Andre A, Abirami S, Jiahuli W et al (2016) The corneal fibrosis response to epithelial-stromal injury. Exp Eye Res 142:110-118

3. Alexander V, Mehrnoosh S (2015) Progress in corneal wound healing. Prog Retin Eye Res 49:17-45

4. Yureeda Q, Gilbert W, Bryan M et al. Corneal transparency: genesis, maintenance and dysfunction. 2010;81:198-210

5. Arsen A, Dilek I, Sibel P et al (2007) Does blunt ocular trauma induce corneal astigmatism? Cornea 26:539-542

6. Alexandra J, Brian K, Heuy-Ching W et al (2020) Multidrug-Resistant Organisms from Ophthalmic Cultures: Antibiotic Resistance and Visual Acuity. Mil Med 185:e1002-e1007

7. McLeod S, LaBree L, Tayyanipour R et al (1995) The importance of initial management in the treatment of severe infectious corneal ulcers. Ophthalmology 102:1943-1948

8. Anita R, Shaffie B, Prabhu V et al (2018) Diagnostic Evaluation of Co-Occurrence of Acanthamoeba and Fungi in Keratitis: A Preliminary Report. Cornea 37:227-234

9. Noopur G, Radhika T (2008) Investigative modalities in infectious Keratitis. Indian J Ophthalmol 56:209-213

10. Jason W, Heather C, Fiona C et al (2013) Dressings for superficial and partial thickness burns. Cochrane Database Syst Rev 2013:CD002106

11. Daniel B, Ashkan J (2008) Pressure ulcers: prevention, evaluation, and management. Am Fam Physician 78:1186-1194

12. Mingyue $Z$, Chenglei T, Tingjun $F$ et al (2019) Fibronectin regulates the self-renewal of rabbit limbal epithelial stem cells by stimulating the Wnt11/Fzd7/ROCK non-canonical Wnt pathway. Exp Eye Res 185:107681

13. Zhao M, Dick A, Forrester $J$ et al (1999) Electric field-directed cell motility involves up-regulated expression and asymmetric redistribution of the epidermal growth factor receptors and is enhanced by fibronectin and laminin. Mol Biol Cell 10:1259-1276

14. Maldonado B, Furcht L (1995) Epidermal growth factor stimulates integrin-mediated cell migration of cultured human corneal epithelial cells on fibronectin and arginine-glycine-aspartic acid peptide. 
Invest Ophthalmol Vis Sci 36:2120-2126

15. Mochizuki H, Yamada M, Hatou S et al (2009) Turnover rate of tear-film lipid layer determined by fluorophotometry. Br J Ophthalmol 93:1535-1538

16. Luigina S, Trefford S, Stephanie V et al (2004) Tear turnover rate is reduced in patients with symptomatic dry eye. Cont Lens Anterior Eye 27:15-20

\section{Figures}




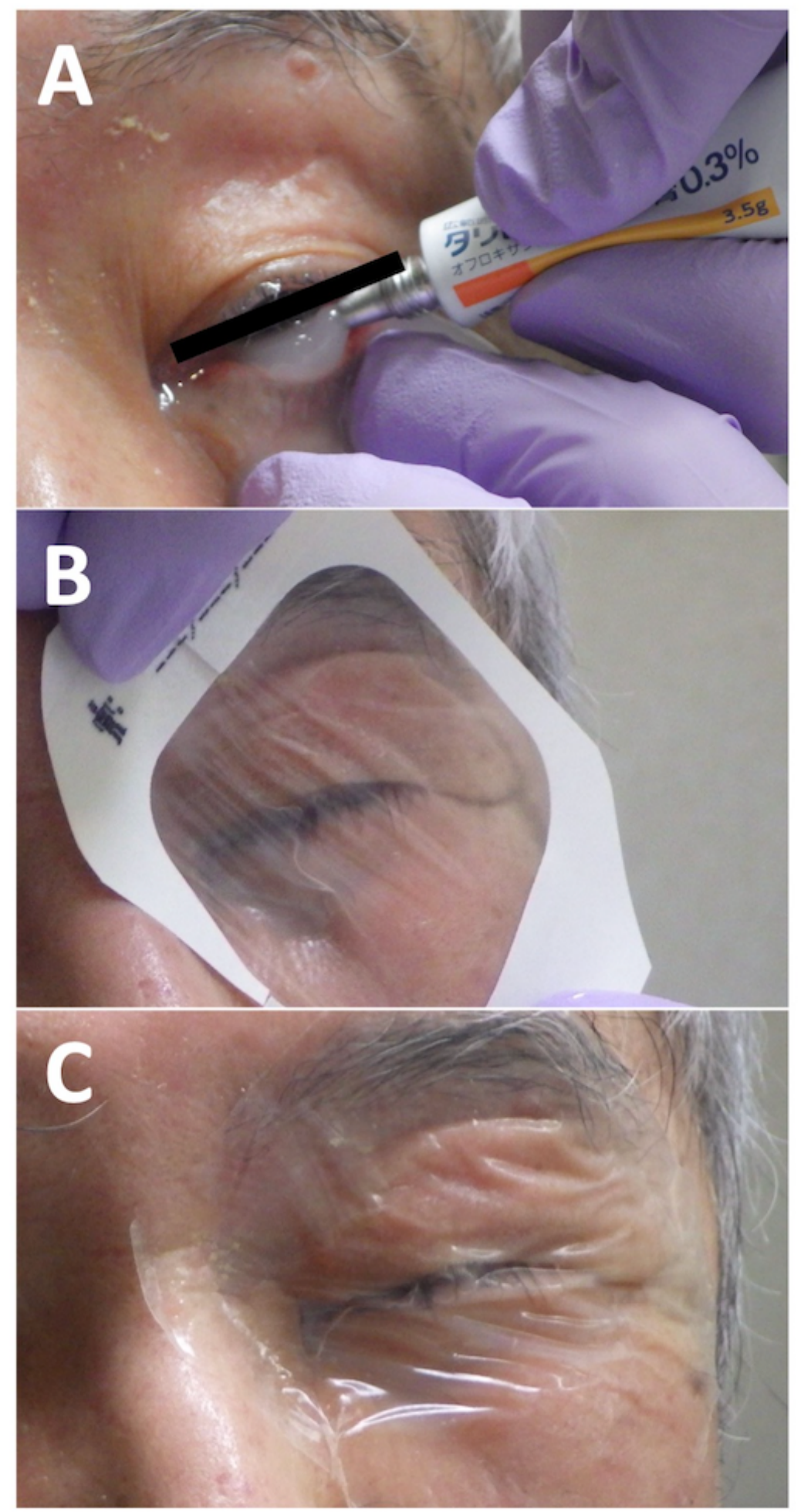

\section{Figure 1}

A: Approximately $0.5 \mathrm{~g}$ appropriate eye ointment is applied to the lower eyelid. B: Tegaderm transparent dressing $(3 \mathrm{M})$ is affixed over and around the eye. C: The eye is closed under Tegaderm transparent dressing $(3 M)$. 


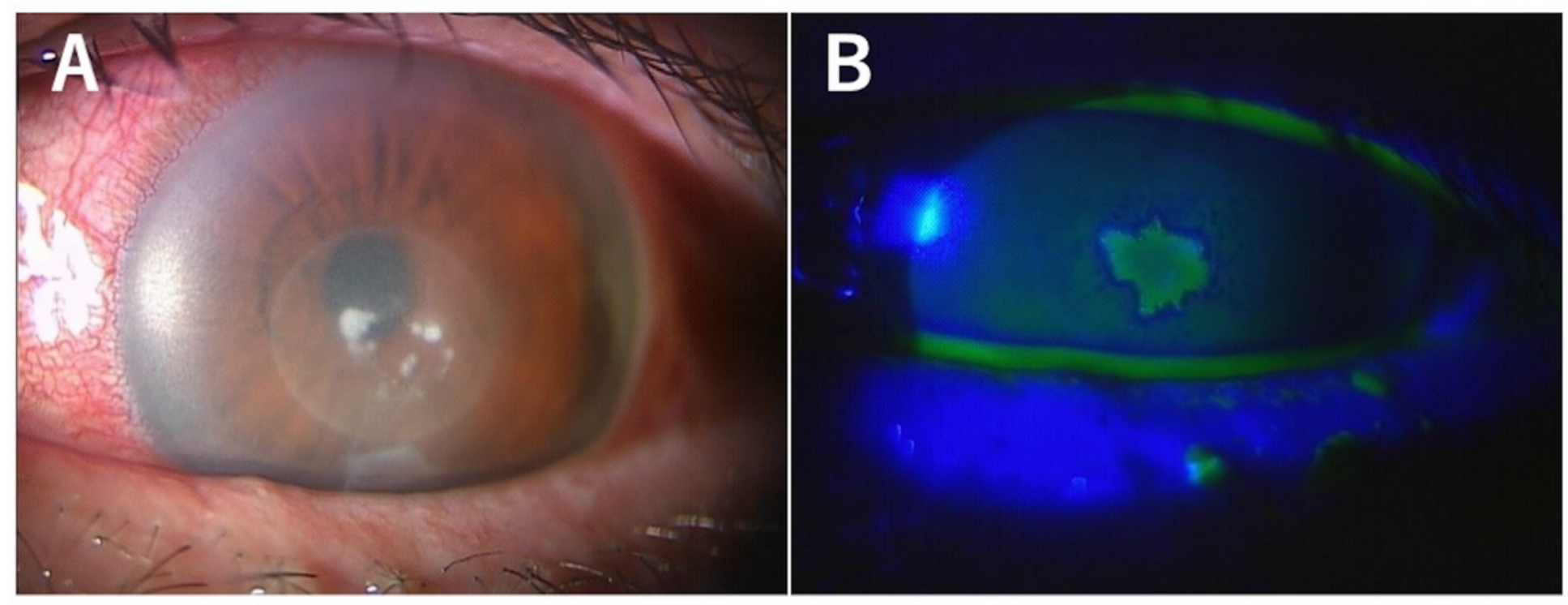

\section{Figure 2}

Central corneal ring ulcer with opacity. A: Anterior segment photograph. B: Photograph showing fluorescein staining.

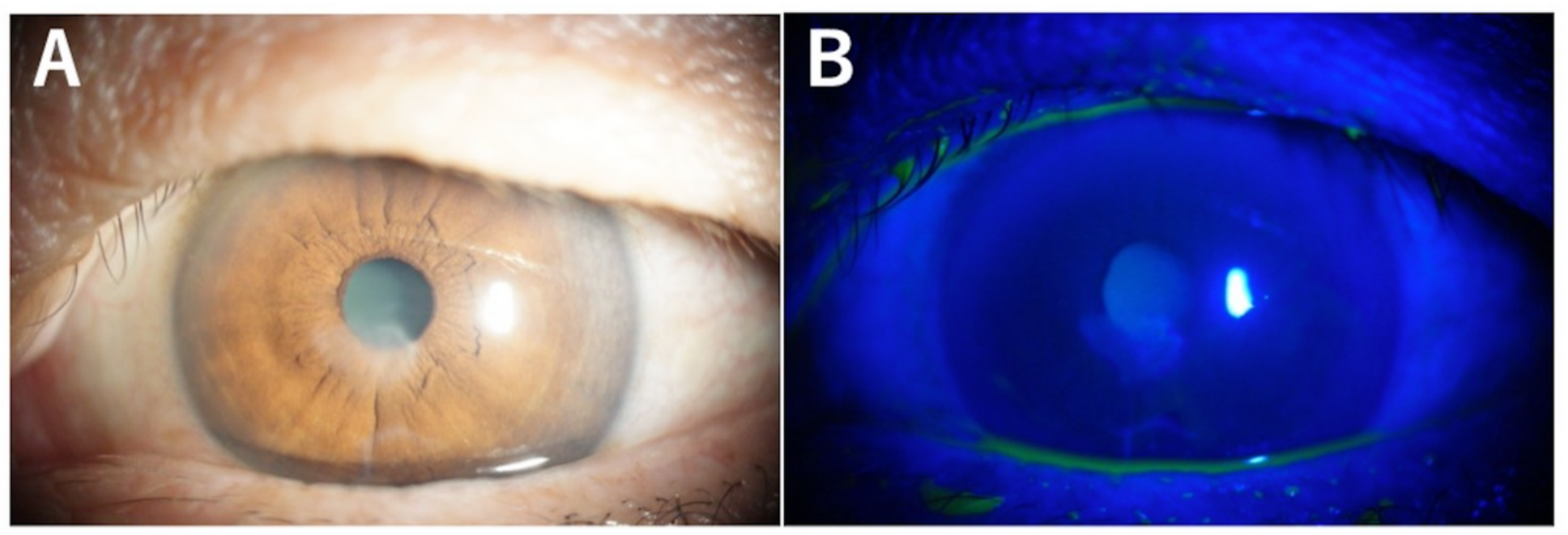

\section{Figure 3}

The ulcer and edema disappeared, although opacity remained in the central cornea. This resolved without recurrence even after discontinuation of AWT. A: Anterior segment photograph. B: Photograph showing fluorescein staining. 